\title{
Efficient Oxidative Removal of Organic Pollutants by Ordered Mesoporous Carbon-Supported Cobalt Phthalocyanine
}

\author{
Yi Chen, Yan Gu, Nan Li, Wangyang Lu, and Wenxing Chen \\ National Engineering Lab for Textile Fiber Materials \& Processing Technology (Zhejiang), \\ Zhejiang Sci-Tech University, Hangzhou 310018, China
}

Correspondence should be addressed to Nan Li; linan@zstu.edu.cn

Received 13 June 2016; Accepted 20 September 2016

Academic Editor: Andrew R. Barron

Copyright (C) 2016 Yi Chen et al. This is an open access article distributed under the Creative Commons Attribution License, which permits unrestricted use, distribution, and reproduction in any medium, provided the original work is properly cited.

Ordered mesoporous carbon (OMC) materials have received attention for use as supports in highly efficient catalytic systems because of their excellent properties. We used epoxy compound 2,3-epoxypropyl trimethylammonium chloride (EPTAC) to modify cobalt tetraaminophthalocyanine (CoTAPc) and obtained a novel catalyst (OMC-CoTAPc-EPTAC) based on OMC-bonded CoTAPc-EPTAC that could oxidize Acid Red 1 (AR1) dyes by hydrogen peroxide $\left(\mathrm{H}_{2} \mathrm{O}_{2}\right)$ activation under neutral conditions. OMC enhanced the catalytic performance of OMC-CoTAPc-EPTAC, which resulted in the combined high catalytic activity and high stability. Because of its large surface area and tunable pore texture, OMC has high substrate accessibility, and the modification of the catalyst with EPTAC could promote adsorption of the target substrate into OMC, which achieved the aim of in situ catalytic oxidation with enrichment of the target substrate and improved the catalytic efficiency significantly. Electron paramagnetic resonance spin-trap experiments confirmed that the OMC-CoTAPc-EPTAC/ $\mathrm{H}_{2} \mathrm{O}_{2}$ system had a nonradical catalytic mechanism, and the high-valent cobalt-oxo intermediates and generated holes were speculated to act as dominant oxidation species for the catalytic degradation of AR1. These results demonstrated a new strategy for the elimination of low-concentration organic pollutants.

\section{Introduction}

Ordered mesoporous carbon (OMC) materials have received considerable attention because of their stable physical and chemical properties and excellent structural characteristics (i.e., large surface area, tunable pore texture, and uniform pore size) [1-5], which shows great promise for application in catalysis, pollutant adsorption, energy storage, and electrochemistry [6-12]. With their porous interfacial structure and controlled mesopore size, OMC materials exhibit great potential for the adsorption of different types of substrates, especially for bulky molecules, and also provide ample space for substrate diffusion $[13,14]$. The larger pore size and ordered three-dimensional open channels of the OMC materials provide them with obvious advantages as catalyst carriers for substrate conversion and the reduction of mass transfer limitations.

Metallophthalocyanines (MPcs) have been studied as catalysts in many applications [15-19], for example, sulfonated cobalt phthalocyanines have been shown to perform catalytic oxidation of mercaptans to remove most sulfur from petrol in the Merox process [20], which results because their structure correlates with porphyrin complexes and also because they are readily available on a large scale and are inexpensive. MPcs are fascinating catalysts, with the main focus being on catalytic oxidation for various organic pollutants [2124]. In general, these catalytic reactions with MPcs occur through electron exchange with coordination between the central metal ion and the reactant. The highly conjugated macrocyclic structure of MPcs as an electron donor can result in electron loss after oxidation and as an electron acceptor can gain electrons after reduction. Several factors, such as substituents and supports, are considered to influence the MPcs catalytic properties. In addition, to achieve their separation and recycle, it is important to choose an appropriate support to immobilize MPcs, where the catalyst activity can be maintained or improved and secondary pollution can be controlled. In our previous work, we found that cellulosic fiber-supported MPcs could eliminate organic dyes [25] but were less efficient for the oxidative removal of 
phenols, possibly because of the poor adsorption of phenols or the low catalytic activity. Some studies also indicated that the introduction of carbon materials could lead to significant enhancement in MPcs activity because of the electron interaction between carbon materials and MPcs [26, 27]. For instance, carbon nanotubes (CNTs) with a special $\mathrm{sp}^{2}$ hybridized structure could enhance the catalytic activity of cobalt phthalocyanine in activating hydrogen peroxide $\left(\mathrm{H}_{2} \mathrm{O}_{2}\right)[28,29]$; activated carbon fiber could improve the cobalt phthalocyanine catalytic performance toward phenol oxidation [30] but appears ineffectual for larger substrates.

Therefore, OMC may be an ideal candidate to support MPcs with a combined high substrate accessibility and enhanced catalyst activity. Here, we used OMC to bond cobalt tetraaminophthalocyanine (CoTAPc) modified by the epoxy compound, 2,3-epoxypropyl trimethylammonium chloride (EPTAC), and obtained a new catalyst with positively charged quaternary ammonium salt chain (OMC-CoTAPc-EPTAC), where the EPTAC led to a highly efficient adsorption for target substrates, especially for negatively charged substrates. Because of requirements for environmentally friendly development, the clean oxidant $\mathrm{H}_{2} \mathrm{O}_{2}$ was used to construct a catalytic oxidation system, OMC-CoTAPc-EPTAC/ $\mathrm{H}_{2} \mathrm{O}_{2}$. The system accomplished the aim of in situ catalytic oxidation with the enrichment of target substrate, which improves the catalytic efficiency significantly. This system is expected to be applied in the treatment of organic pollutants, especially for microcontaminants that are difficult to remove. In the OMCCoTAPc-EPTAC/ $\mathrm{H}_{2} \mathrm{O}_{2}$ system, enhanced catalytic performance was evidenced by the oxidative degradation of the dye Acid Red 1 (AR1). The electron paramagnetic resonance (EPR) spin-trap technique was used to determine the possible mechanism for $\mathrm{H}_{2} \mathrm{O}_{2}$ activation by OMC-CoTAPc-EPTAC. Key factors that are likely to influence the oxidation process, such as temperature and $\mathrm{H}_{2} \mathrm{O}_{2}$ concentration, were explored, and the stability of the catalytic system was discussed.

\section{Experimental}

2.1. Materials and Reagents. AR1 and the spin-trapping reagent 5,5-dimethyl-1-pyrroline $\mathrm{N}$-oxide (DMPO) were obtained from Tokyo Chemical Industry Co., Ltd. Cobalt chloride hexahydrate, urea, and hydrogen peroxide $(9.7 \mathrm{M}$, Sinopharm Chemical Reagent Co., Ltd.) were used as analytical reagents. Doubly distilled water was used throughout the AR1 removal experiments. All other solvents were of spectrometric grade.

2.2. Catalyst Preparation. OMC was synthesized from resorcinol, F127, formaldehyde, ethanol, and $\mathrm{HCl}$ [31], and CoTAPc was synthesized from 4-nitrophthalic acid, cobalt chloride hexahydrate, and urea [32]. CoTAPc $(0.05 \mathrm{~g})$ was dissolved in dimethyl sulfoxide solution. The solution was stirred vigorously and kept at $85^{\circ} \mathrm{C}$ for $30 \mathrm{~min}$ in an oil bath before $0.02 \mathrm{~g}$ of EPTAC $\left(n_{\mathrm{EPTAC}}: n_{\mathrm{CoTAPc}}=1.5: 1\right)$ was added. After reaction for $5 \mathrm{~h}, \mathrm{OMC}(1 \mathrm{~g})$ was added to the reaction solution, stirring constantly, and then isopropyl nitrite was added to the reaction at $85^{\circ} \mathrm{C}$ for $12 \mathrm{~h}$. The product was purified by centrifugation and washing with ultrapure water several times. OMC-CoTAPc-EPTAC was obtained after drying. This method was inspired by [33], and the synthesis is shown in Scheme 1 (the part functionalization of the amino groups on CoTAPc, taking the 1:4 molar ratio functionalization of the amino groups as the example).

2.3. Catalyst Characterizations. Ultraviolet- (UV-) visible (Vis) absorption spectra were acquired at ambient temperature on a Hitachi U-3100 spectrophotometer, and spectra were recorded from 400 to $800 \mathrm{~nm}$. The porous structure of the samples was observed by transmission electron microscopy (TEM) using a JEOL JEM-2010 microscope.

Nitrogen $\left(\mathrm{N}_{2}\right)$ adsorption/desorption isotherms were obtained at $-196^{\circ} \mathrm{C}$ using a Micromeritics ASAP 2020 apparatus. Prior to the measurement, samples were degassed at $180^{\circ} \mathrm{C}$ for at least $6 \mathrm{~h}$. To calculate the surface area, the Brunauer-Emmett-Teller (BET) model was applied for $0.06 \leq$ $P / P_{0} \leq 0.30$. The mesoporous volume was calculated from the amount of nitrogen adsorbed at a relative pressure of 0.95 , and the pore size distribution was evaluated using the BarrettJoyner-Halenda model.

Powder X-ray diffraction patterns (XRD) were measured on an HP DX-2700 diffractometer operated at $40 \mathrm{kV}$ and $40 \mathrm{~mA}$ and equipped with a graphite monochromator using $\mathrm{Cu}-\mathrm{Ka}$ radiation. Measurements were achieved for $0.1-80^{\circ} 2 \theta$ with steps of $0.02^{\circ}$.

Thermogravimetric analyses (TGA) were carried out on a TG Mettler Toledo STARe apparatus, with a heating rate of $20^{\circ} \mathrm{C} / \mathrm{min}$ from 25 to $800^{\circ} \mathrm{C}$ in $\mathrm{N}_{2}$ to determine the thermal stability of the samples.

The cobalt content in OMC-CoTAPc-EPTAC was confirmed to be $49.42 \mu \mathrm{mol} / \mathrm{g}$ by microwave-assisted digestionflame atomic absorption spectrometry (Thermo Sollar M6).

2.4. Catalytic Oxidation. The catalytic activity of OMCCoTAPc-EPTAC in activating $\mathrm{H}_{2} \mathrm{O}_{2}$ in aqueous solution was investigated by the oxidative degradation of AR1. The initial concentration of AR1 was $0.05 \mathrm{mM}$, and oxidation was initiated when $\mathrm{H}_{2} \mathrm{O}_{2}(15 \mathrm{mM})$ was added into the solution in the presence of OMC-CoTAPc-EPTAC $(0.4 \mathrm{~g} / \mathrm{L})$. All reactions were performed in a container agitated with a magnetic stirrer and heated in a temperature-controlled water bath under neutral conditions. At given times, samples were analyzed with a UV-Vis spectrometer by measuring the removal of AR1 at the maximal wavelength. Stability and reusability were addressed through a cycle of five continuous experiments. For every run, a specific concentration of AR1 was added into the reaction system to maintain the initial $0.05 \mathrm{mM}$ concentration, and $5 \mathrm{mM} \mathrm{H}_{2} \mathrm{O}_{2}$ was added to each cycle starting from the next cycle. The catalytic mechanism was analyzed by EPR, and the EPR signals of radicals trapped by DMPO were detected on a Bruker A300 spectrometer.

\section{Results and Discussion}

3.1. Characterization of OMC-CoTAPc-EPTAC. The UV-Vis absorption spectra of CoTAPc, OMC, and OMC-CoTAPcEPTAC are shown in Figure 1. The CoTAPc spectrum shows a sharp absorption peak at $705 \mathrm{~nm}$ and a weaker absorption 


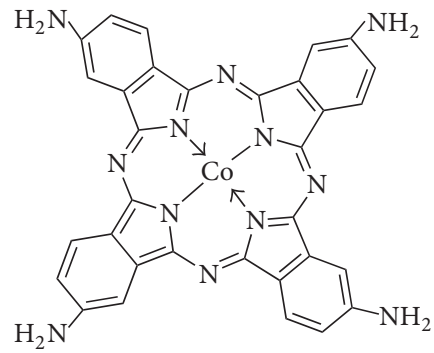
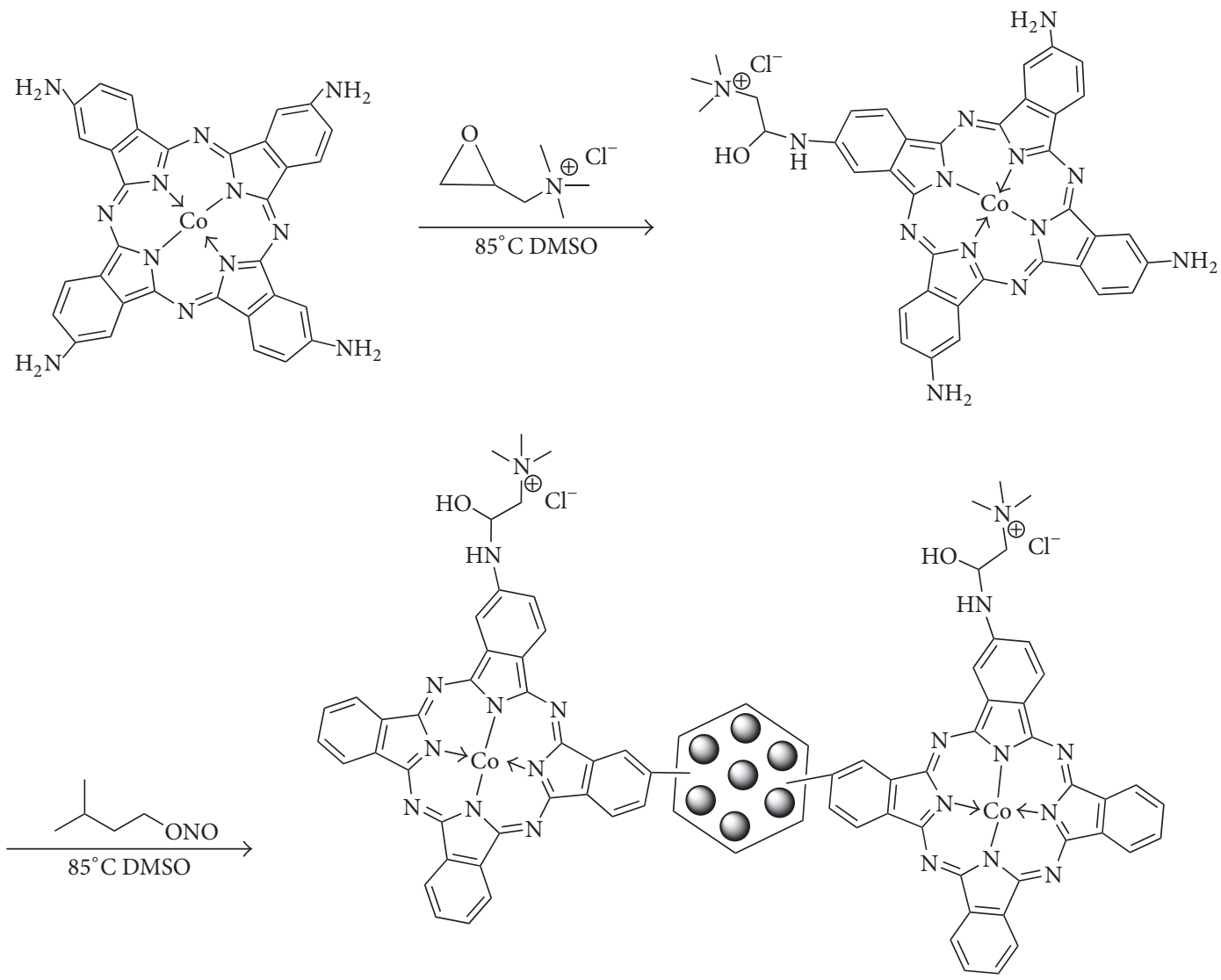

SCHEME 1: Synthesis of OMC-CoTAPc-EPTAC.

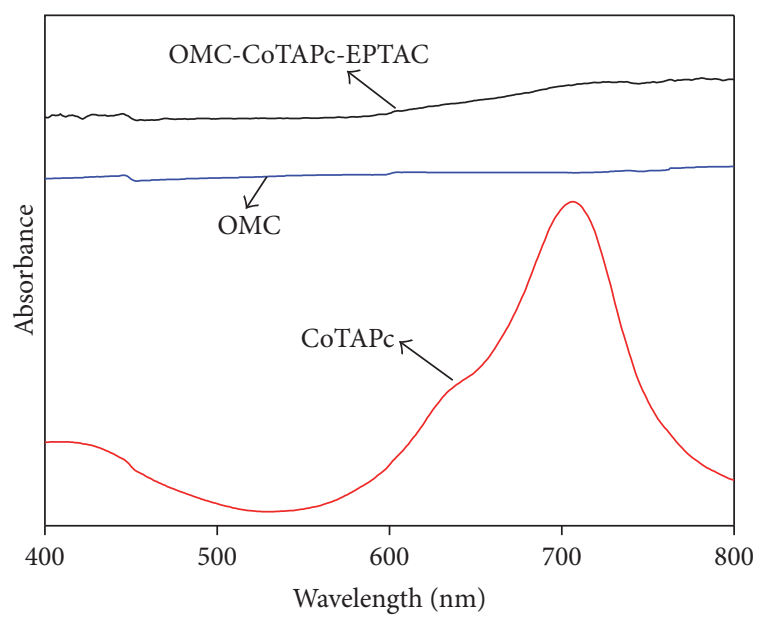

FIGURE 1: UV-Vis absorption spectra for OMC, CoTAPc, and OMCCoTAPc-EPTAC (in DMF).

peak at $412 \mathrm{~nm}$ because of the strong electron-donating property of the amino group on the CoTAPc ring. The spectrum does not show any absorption peaks at $400-800 \mathrm{~nm}$ for OMC, whereas the OMC-CoTAPc-EPTAC spectrum shows a broad absorption peak at $600-750 \mathrm{~nm}$, which may be explained by the strong electronic interaction between CoTAPc and OMC.
Therefore, we conclude that CoTAPc was supported on the OMC.

The textural properties of OMC and OMC-CoTAPcEPTAC were investigated using high-resolution TEM and $\mathrm{N}_{2}$ absorption. The OMC surface morphology before and after modification with CoTAPc-EPTAC was studied by TEM (Figure 2). The compounds possessed a well-defined ordered mesoporous structure with uniform size, and the ordered mesostructure of OMC was rarely destroyed after modification with CoTAPc-EPTAC. The pore diameter is not visible in the TEM micrographs because of the superimposition of the three-dimensional mesopore system [34-36]. $\mathrm{N}_{2}$ adsorption/desorption isotherms were used to explore the pore structure and adsorption capacity of the samples further. Figure 3(a) showed an obvious increase in $\mathrm{N}_{2}$ uptake especially in the low-pressure region, which indicates the formation of highly porous carbon. A typical type IV isotherm with $\mathrm{H} 2$ hysteresis loops and a significant capillary condensation step for relative pressures of $0.45-0.8$ was obtained, which suggests that the mesopore size was almost identical as shown by the corresponding pore size distribution analysis (Figure 3(b)). This confirmed that OMC and OMC-CoTAPcEPTAC had a narrow $\sim 6 \mathrm{~nm}$ pore size distribution in the mesopore range. According to the calculation results of the $\mathrm{N}_{2}$ adsorption/desorption isotherms, the BET surface area of $\mathrm{OMC}$ is $636 \mathrm{~m}^{2} / \mathrm{g}$, which decreased to $530 \mathrm{~m}^{2} / \mathrm{g}$ after bonding cobalt tetraaminophthalocyanine. This could be explained by 


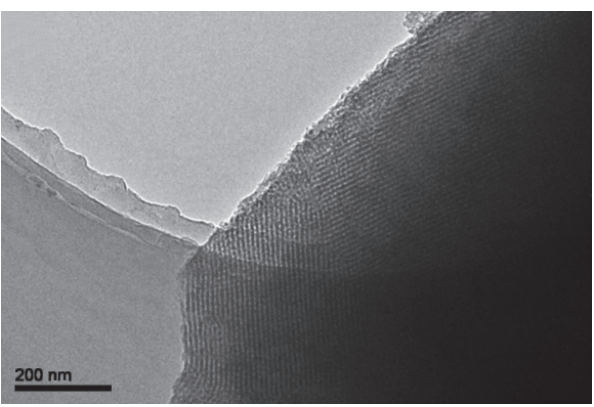

(a)

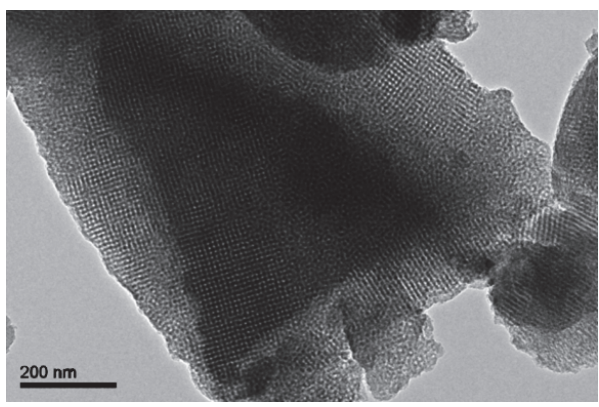

(c)

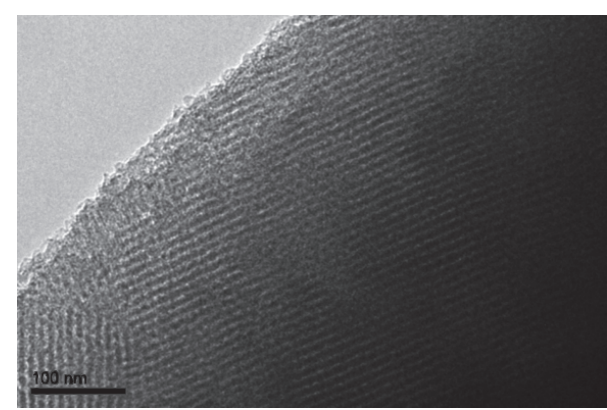

(b)

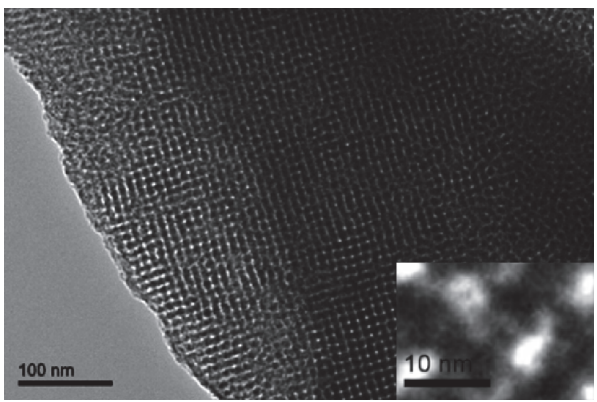

(d)

FIGURE 2: (a) and (b) TEM image of OMC calcined at $850^{\circ} \mathrm{C}$. (c) and (d) TEM image of OMC-CoTAPc-EPTAC.

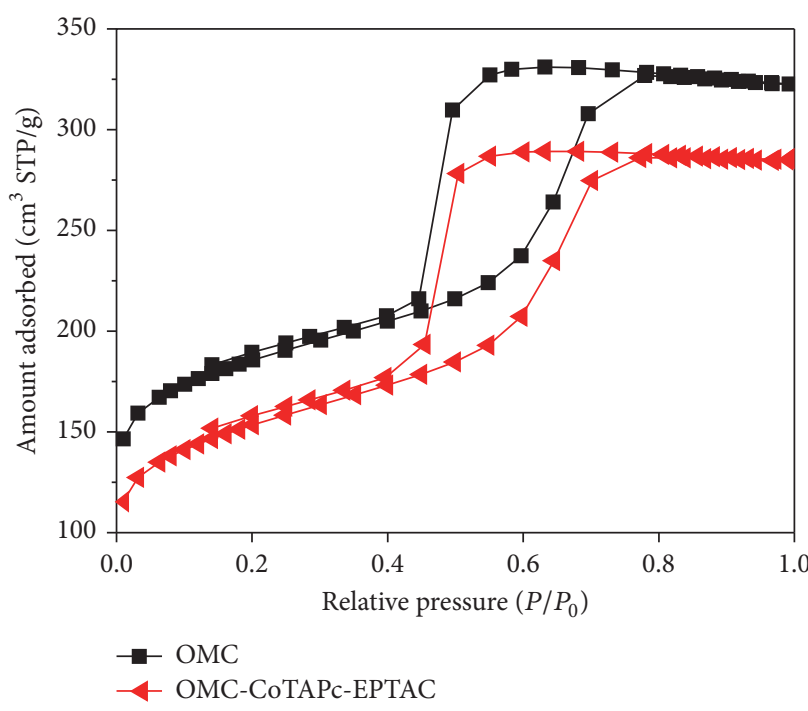

(a)

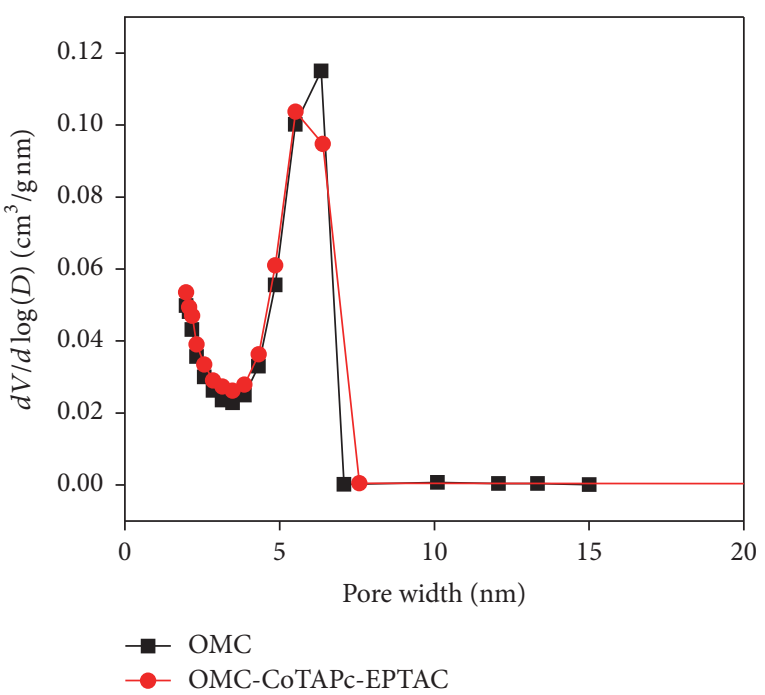

(b)

FIgURE 3: (a) $\mathrm{N}_{2}$ sorption isotherm and (b) pore size distribution of OMC and OMC-CoTAPc-EPTAC.

the fact that the interior OMC space was occupied partially by the CoTAPc-EPTAC. The ordered OMC-CoTAPc-EPTAC mesoporous structure was maintained and the OMC support exhibited relatively high adsorption capacity. This was consistent with the TEM observation.

Additional structural information was obtained from the powder XRD analysis (Figure 4). OMC has an XRD pattern with a sharp diffraction peak at $2 \theta=24^{\circ}$ and weaker peaks at $2 \theta=45^{\circ}$ that correspond to the (002) and (101) planes of carbon, respectively [37], which means that OMC had some ordered graphite crystallites after high-temperature carbonization. Figure 4 also shows that the diffraction peak of CoTAPc was centered at $2 \theta=28^{\circ}$. The diffraction pattern of OMC-CoTAPc-EPTAC was similar to that of OMC, and the supported catalyst had no noticeable effect on the diffraction peaks, except that they were slightly weaker, probably because the crystalline structure of the OMC support was partially destroyed. These observations suggest that the CoTAPc had 


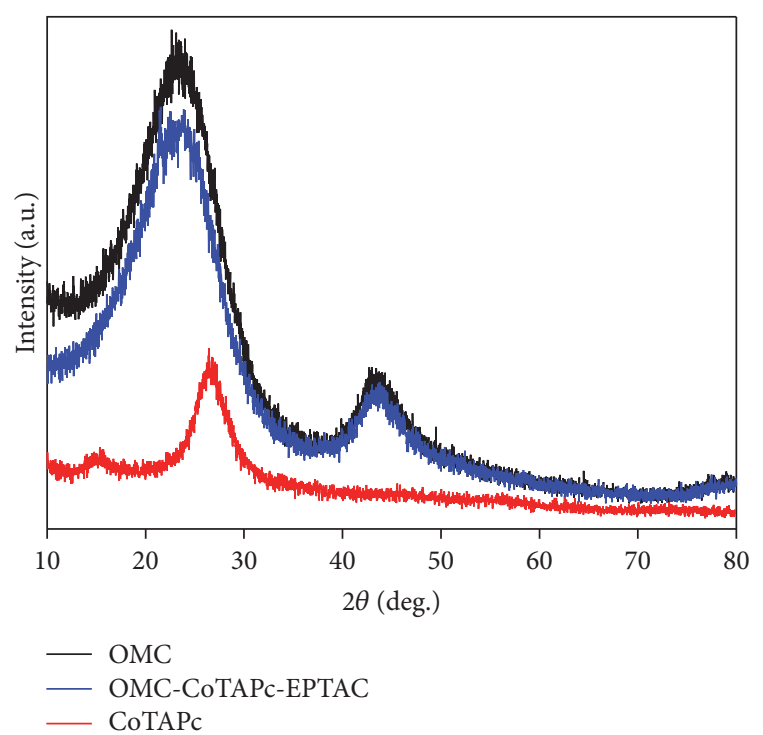

Figure 4: XRD patterns for OMC, CoTAPc, and OMC-CoTAPcEPTAC.

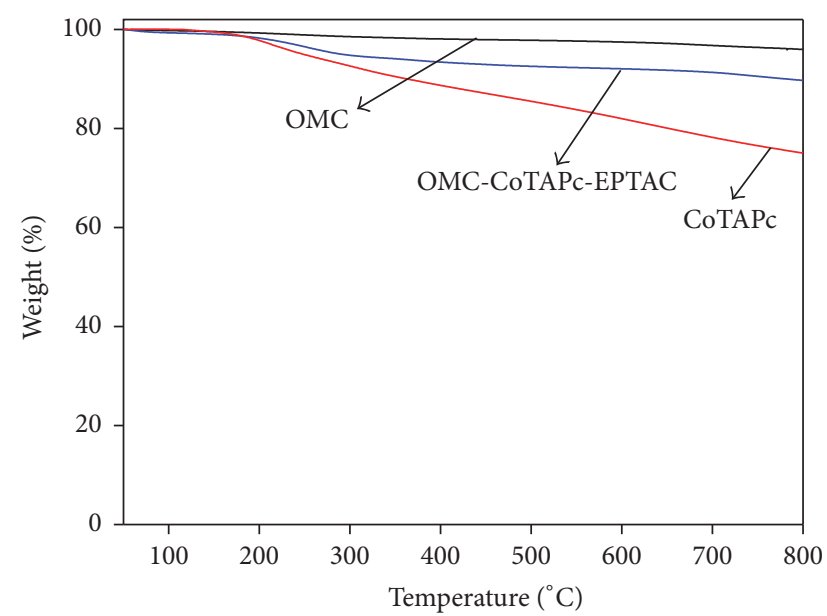

Figure 5: TGA for OMC, CoTAPc, and OMC-CoTAPc-EPTAC.

little influence on the OMC structure. To evaluate the thermal stability of the catalyst, TGA was carried out in $\mathrm{N}_{2}$ (Figure 5). OMC has an excellent thermal stability from 50 to $800^{\circ} \mathrm{C}$. CoTAPc mass loss starts to occur at $200^{\circ} \mathrm{C}$, and eventually some thermal decomposition will result. The mass loss curve of OMC-CoTAPc-EPTAC lay between that of OMC and CoTAPc, which means that the thermal stability of CoTAPc improved by introducing $\mathrm{OMC}$ as the support.

3.2. Oxidative Degradation of AR1. To investigate the catalytic performance of OMC-CoTAPc-EPTAC, AR1 in aqueous solution was used as the model for catalytic oxidation. Figure 6 shows that AR1 cannot be oxidized directly with $\mathrm{H}_{2} \mathrm{O}_{2}$ alone. In our previous work, we found that even in the presence of $\mathrm{H}_{2} \mathrm{O}_{2}$ and CoTAPc, almost no removal of target dyes resulted [28]. OMC-supported CoTAPc (OMCCoTAPc) could only adsorb dyes fractionally, which resulted

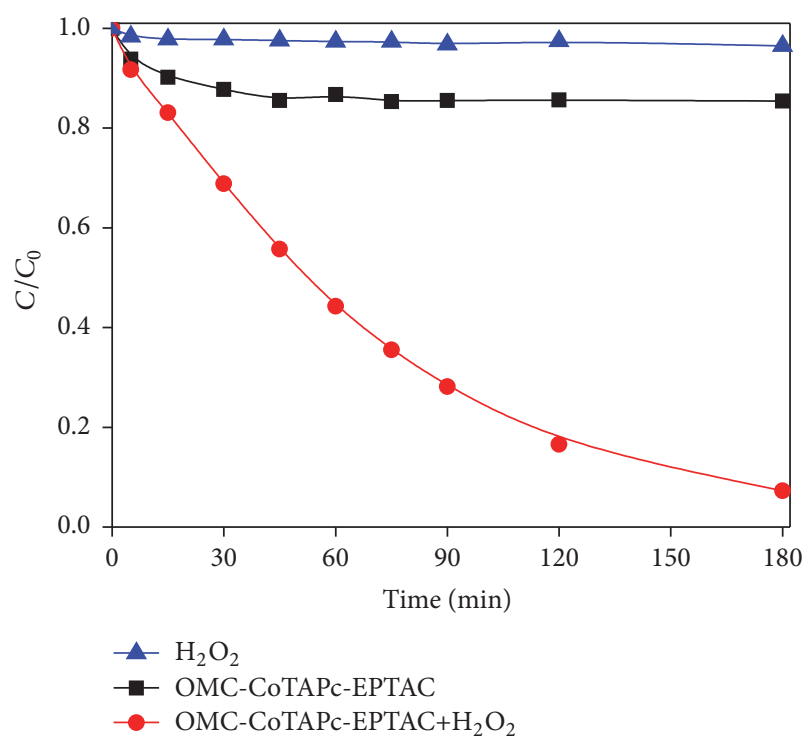

FIGURE 6: Concentration changes of AR1 $(0.05 \mathrm{mM})$ under different conditions $\left([\mathrm{OMC}-\mathrm{CoTAPc}-\mathrm{EPTAC}]=0.4 \mathrm{~g} / \mathrm{L},\left[\mathrm{H}_{2} \mathrm{O}_{2}\right]=15 \mathrm{mM}\right.$, $\mathrm{pH} 7$, and $\left.50^{\circ} \mathrm{C}\right)$.

in a low oxidative removal rate of dyes with $\mathrm{H}_{2} \mathrm{O}_{2}$ [38]. Figure 6 shows that in the presence of OMC-CoTAPc-EPTAC alone, a clear decrease in AR1 resulted and the removal rate remained constant because the adsorption process attained equilibrium in a short time. The AR1 concentration decreased significantly when AR1 was exposed to OMC-CoTAPcEPTAC and $\mathrm{H}_{2} \mathrm{O}_{2}$ together. These results suggest that the introduction of EPTAC into the catalyst induced a positive effect on improving the oxidative degradation efficiency of AR1 by facilitating the adsorption of dye into OMC. According to the previously described experiments, we infer that the removal of AR1 in the OMC-CoTAPc-EPTAC/ $\mathrm{H}_{2} \mathrm{O}_{2}$ system included two processes: adsorption of AR1 into the OMCCoTAPc-EPTAC and catalytic oxidation of the adsorbed AR1. Figure 7 shows that AR1 adsorption reached equilibrium in a short time without $\mathrm{H}_{2} \mathrm{O}_{2}$. Its concentration decreased significantly once $\mathrm{H}_{2} \mathrm{O}_{2}$ was added into the adsorption equilibrium system, which indicates that rapid and efficient oxidation of AR1 could be achieved when $\mathrm{H}_{2} \mathrm{O}_{2}$ was present in the reaction system and the adsorbed AR1 consumption was replenished continuously from AR1 in the bulk solution until the removal was complete. It is a great achievement that dyes could be enriched rapidly into OMC-CoTAPc-EPTAC and be decomposed in situ. Therefore, we conclude that the OMC-CoTAPc-EPTAC had high-level catalytic activity for AR1 oxidation, and OMC with a large adsorption capacity played a critical role during the degradation.

Temperature is an important factor that influences the catalytic reaction rate. To investigate the effect of reaction temperature on the OMC-CoTAPc-EPTAC/ $\mathrm{H}_{2} \mathrm{O}_{2}$ catalytic system, the catalytic oxidation of AR1 was carried out at different temperatures with the result presented in Figure 8. In the presence of $\mathrm{H}_{2} \mathrm{O}_{2}$, OMC-CoTAPc-EPTAC could catalyze the oxidative removal of AR1 from 25 to $75^{\circ} \mathrm{C}$, and the 


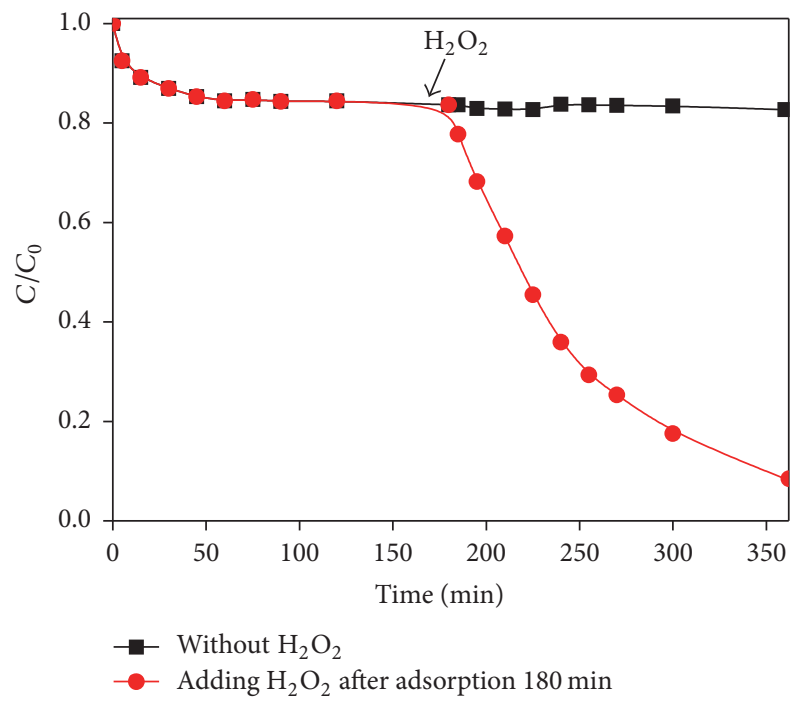

FIGURE 7: Concentration changes of AR1 ( $0.05 \mathrm{mM})$ after adsorption equilibrium with or without $\mathrm{H}_{2} \mathrm{O}_{2}$ ([OMC-CoTAPc-EPTAC] $=$ $0.4 \mathrm{~g} / \mathrm{L},\left[\mathrm{H}_{2} \mathrm{O}_{2}\right]=15 \mathrm{mM}, \mathrm{pH} 7$, and $\left.50^{\circ} \mathrm{C}\right)$.

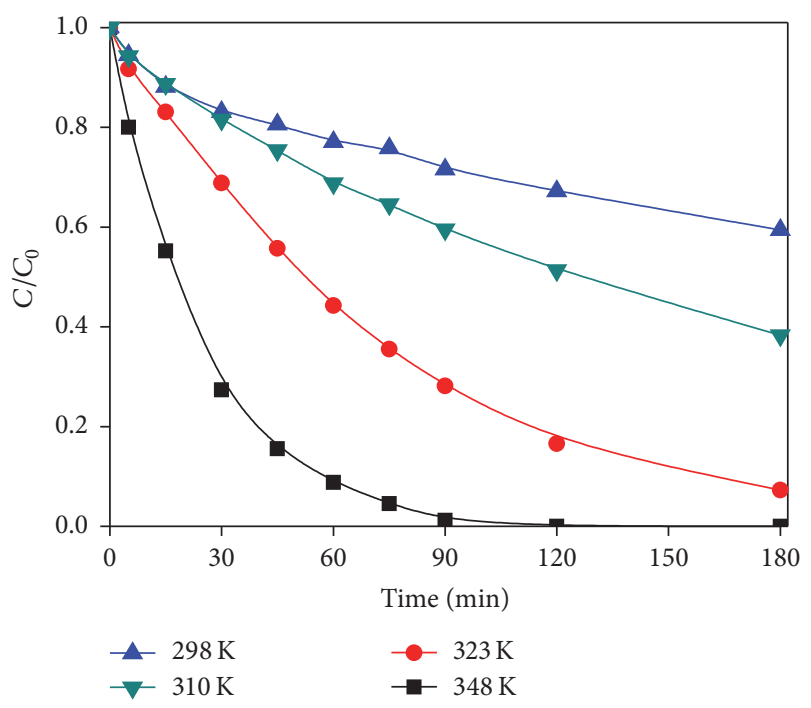

FIGURE 8: Effect of temperature on catalytic oxidation of AR1 $(0.05 \mathrm{mM})\left([\mathrm{OMC}-\right.$ CoTAPc-EPTAC $]=0.4 \mathrm{~g} / \mathrm{L},\left[\mathrm{H}_{2} \mathrm{O}_{2}\right]=15 \mathrm{mM}$, and $\mathrm{pH} 7)$.

removal rate of AR1 increased as the reaction temperature increased. This may occur because the thermal energy of the AR1 molecules increased with increase in temperature, which resulted in a higher molecular heat movement speed. It was conducive to dye diffusion into the catalytic active sites, and enhanced the reaction rate. Based on the time courses of $\ln \left(C_{0} / C\right)$ at four different temperatures, an Arrhenius plot of $\ln k-1 / T$ is shown in Figure 9, and the activation energy of the oxidative degradation of AR1 in the OMC-CoTAPcEPTAC $/ \mathrm{H}_{2} \mathrm{O}_{2}$ system was $49.8 \mathrm{~kJ} / \mathrm{mol}$ as calculated from the slope of the straight line. In comparison with common catalytic systems $[39,40]$, the system with a lower activation

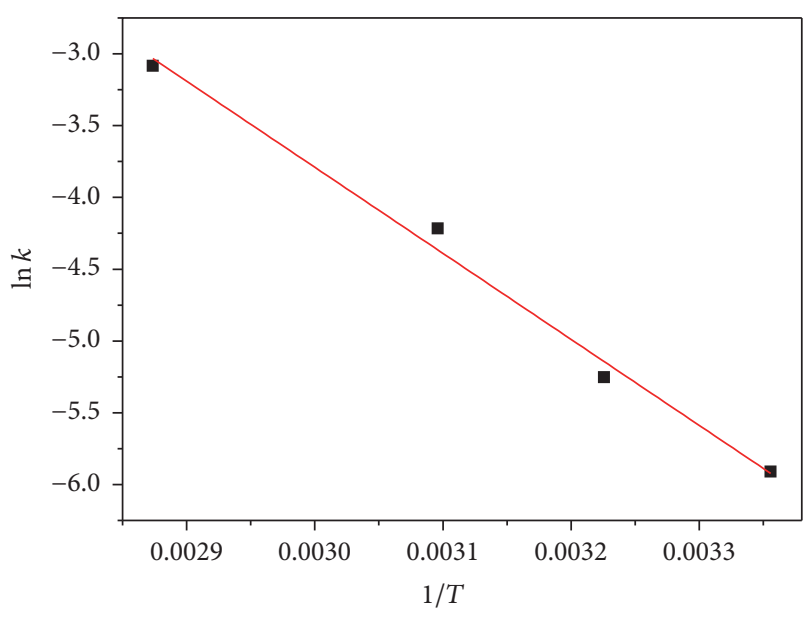

FIGURE 9: Arrhenius plots of $\ln k-1 / T$ with OMC-CoTAPc-EPTAC as catalyst.

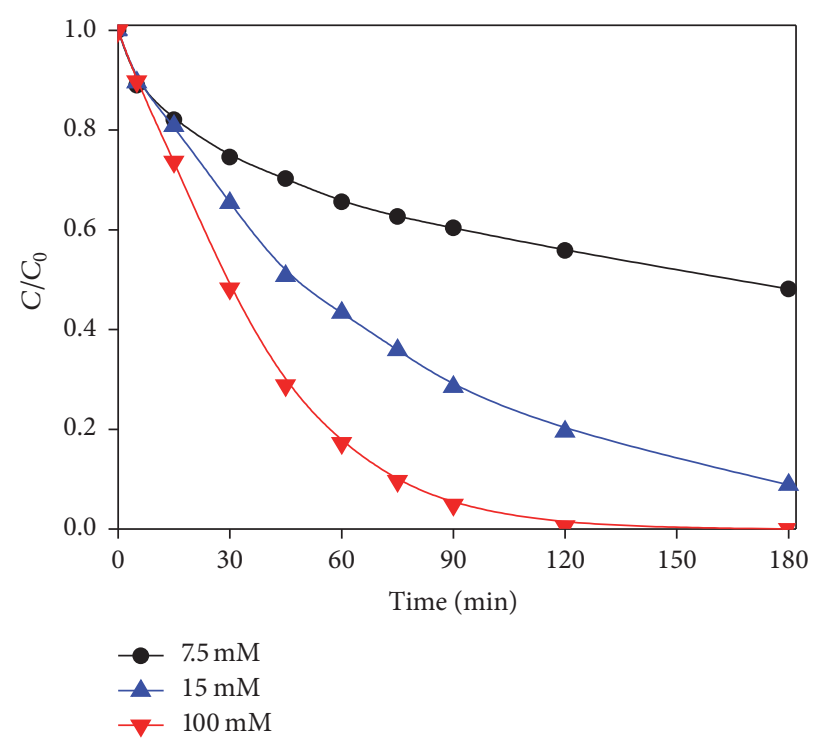

Figure 10: Effect of initial $\mathrm{H}_{2} \mathrm{O}_{2}$ concentration on catalytic oxidation of AR1 $(0.05 \mathrm{mM})([\mathrm{OMC}-\mathrm{CoTAPc}-\mathrm{EPTAC}]=0.4 \mathrm{~g} / \mathrm{L}, \mathrm{pH} 7$, and $\left.50^{\circ} \mathrm{C}\right)$.

energy exhibited a high oxidative removal efficiency for AR1 at high temperature.

$\mathrm{H}_{2} \mathrm{O}_{2}$ is an environmentally friendly reagent and is used in almost all industrial fields. A reduction in $\mathrm{H}_{2} \mathrm{O}_{2}$ dosage is commercially important in chemical reaction processes. The effect of $\mathrm{H}_{2} \mathrm{O}_{2}$ dosage on AR1 removal efficiency was explored by varying the initial $\mathrm{H}_{2} \mathrm{O}_{2}$ concentration from 7.5 to $100 \mathrm{mM}$ with results as shown in Figure 10. An increase in the initial $\mathrm{H}_{2} \mathrm{O}_{2}$ concentration led to a higher rate of AR1 degradation, and when increased above $15 \mathrm{mM}$, the AR1 concentration decreased sharply. Based on the $\mathrm{H}_{2} \mathrm{O}_{2}$ utilization efficiency, a $\mathrm{H}_{2} \mathrm{O}_{2}$ concentration of $15 \mathrm{mM}$ was selected as the optimum $\mathrm{H}_{2} \mathrm{O}_{2}$ dosage. Compared with the singlewalled carbon nanotube-supported CoTAPc (MWCNTsCoTAPc) $/ \mathrm{H}_{2} \mathrm{O}_{2}$ catalytic system reported in our previous 


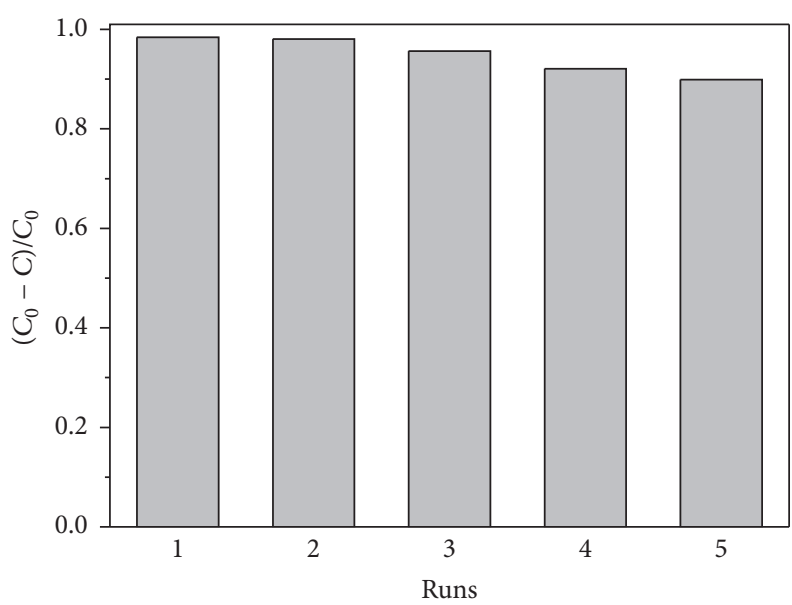

FIgURE 11: Cyclic catalytic oxidation of AR1 (0.05 mM), $5 \mathrm{mM}$ $\mathrm{H}_{2} \mathrm{O}_{2}$ addition at each cycle after the first cycle ([OMC-CoTAPcEPTAC $]=0.4 \mathrm{~g} / \mathrm{L},\left[\mathrm{H}_{2} \mathrm{O}_{2}\right]=15 \mathrm{mM}, \mathrm{pH} 7$, and $\left.50^{\circ} \mathrm{C}\right)$.

paper [28], OMC-CoTAPc-EPTAC had a higher catalytic oxidation efficiency at a lower $\mathrm{H}_{2} \mathrm{O}_{2}$ concentration. This may be because the higher $\mathrm{H}_{2} \mathrm{O}_{2}$ utilization efficiency in the OMCCoTAPc-EPTAC $/ \mathrm{H}_{2} \mathrm{O}_{2}$ catalytic system could be ascribed to a different $\mathrm{H}_{2} \mathrm{O}_{2}$ activation mechanism or MWCNTsCoTAPc served as an interfacial catalyst. Substrate adsorption and oxidation occurred at the MWCNT surface, whereas in the OMC-CoTAPc-EPTAC/ $\mathrm{H}_{2} \mathrm{O}_{2}$ system, a reaction was required for the target substrates and $\mathrm{H}_{2} \mathrm{O}_{2}$ to enter into the interior of the OMC support, which significantly improved the $\mathrm{H}_{2} \mathrm{O}_{2}$ utilization in this catalytic system.

Stability is another significant issue for catalyst use in practical applications. However, to the best of our knowledge, the supported MPcs usually lose partial activity because barriers to oxidation intermediates are generated in the reaction, or they may suffer from autocatalytic decomposition when the catalytic oxidation reaction is dominated by nonselective - $\mathrm{OH}$ [41], which results in a loss of catalytic activity. To study the catalyst stability, it is necessary to carry out continuous cyclic oxidation experiments of AR1 in the catalytic system that involves OMC-CoTAPc-EPTAC and $\mathrm{H}_{2} \mathrm{O}_{2}$. Figure 11 shows that the oxidative removal rate of AR1 was 98\% after the first cycle, and the rate was still high with no obvious decrease after five cycles of reuse. This highlights the excellent catalytic properties and good self-reliance regeneration ability of OMC-CoTAPc-EPTAC in repetitive experiments. These findings show that OMC introduction improves the catalytic activity and stability of CoTAPc and provides a transport channel for degradation products, guaranteeing constant substrate adsorption and oxidation, and achieves catalyst recycling.

3.3. Analysis of Catalytic Mechanism. A catalytic mechanism that is dominated by hydroxyl radical $\left({ }^{\circ} \mathrm{OH}\right)$ oxidation, highvalency metal-oxo species oxidation, or hole oxidation in the activated $\mathrm{H}_{2} \mathrm{O}_{2}$ systems has been reported. To obtain deeper insight into the possible catalytic mechanism of OMCCoTAPc-EPTAC in activating $\mathrm{H}_{2} \mathrm{O}_{2}$, the EPR technique (with DMPO) was used with results shown in Figure 12. Nearly no DMPO- ${ }^{\circ} \mathrm{OH}$ and $\mathrm{DMPO}^{-} \mathrm{OOH}$ signals were detected for AR1 in aqueous or methanol solution, which shows that ${ }^{\circ} \mathrm{OH}$ and ${ }^{\circ} \mathrm{OOH}$ were not major intermediates of the catalytic reaction with OMC-CoTAPc-EPTAC. From the EPR results, we concluded that the catalytic oxidation of AR1 occurred through a nonradical pathway, which was different from the common CoTAPc system [30], where the introduction of $\mathrm{OMC}$ and the direct-bonding method were considered two key factors that influence the reaction channels. In our previous work, high-valent cobalt-oxo intermediates were achieved by using linear alkylbenzene sulfonate as the fifth ligand on cellulosic fiber-boned cobalt phthalocyanine and these displayed good oxidation capacity under neutral conditions [42]. Our earlier study found that the MWCNTs-CoTAPc $/ \mathrm{H}_{2} \mathrm{O}_{2}$ system, which was dominated by hole oxidation, exhibited a relatively high catalytic activity for the oxidative removal of adsorbed dyes at neutral $\mathrm{pH}$ $[28,29]$ and that this corresponds to the catalytic performance of the OMC-CoTAPc-EPTAC/ $\mathrm{H}_{2} \mathrm{O}_{2}$ system. Based on these considerations, we infer that high-valent cobalt-oxo intermediates and generated holes were possible oxidation species during the catalytic reaction, which caused the OMCCoTAPc-EPTAC/ $\mathrm{H}_{2} \mathrm{O}_{2}$ system to exhibit a higher catalytic efficiency and better stability.

The possible pathways for the oxidation of AR1 in this catalytic system are shown in Figure 13. (a) In the OMCCoTAPc-EPTAC $/ \mathrm{H}_{2} \mathrm{O}_{2}$ system, CoTAPc is oxidized by the coordination behavior between $\mathrm{H}_{2} \mathrm{O}_{2}$ and the central cobalt ion. Then the relatively high Fermi level of OMC can provide electrons for the reduction of oxidized CoTAPc and the hole-doped OMC is obtained, achieving the rapid oxidation of the target substrate. (b) At the same time, the center cobalt ion of CoTAPc is coordinated axially by $\mathrm{OOH}^{-}$. The heterolytic cleavage of the $\mathrm{O}-\mathrm{O}$ bond results in the generation of $\mathrm{Co}(\mathrm{IV})=\mathrm{O}$ with a high oxidation ability to the substrate.

\section{Conclusions}

We have obtained a novel catalyst with a positively charged quaternary ammonium salt chain (OMC-CoTAPc-EPTAC) by OMC bonding the CoTAPc-EPTAC. OMC with a tunable pore texture and ordered three-dimensional open channel offered a suitable environment for AR1 accessibility into the active sites. The introduction of OMC into the catalytic system had a "push" effect on the catalytic activity and stability. The higher adsorption capacity of OMC-CoTAPc-EPTAC toward negatively charged substrates was achieved by modifying CoTAPc with EPTAC, which resulted in significant improvement in catalytic efficiency for AR1 oxidation. As expected, the EPR results indicate that the catalytic oxidation of AR1 involved a nonradical mechanism, and the high-valent cobalt-oxo species and generated holes were predicted as appropriate active species. The OMC-CoTAPc-EPTAC/ $\mathrm{H}_{2} \mathrm{O}_{2}$ system displayed a greater catalytic performance at lower $\mathrm{H}_{2} \mathrm{O}_{2}$ dosage and achieved a low cost and high efficiency for practical applications. The combination of adsorption enrichment and in situ catalytic oxidation provides a preferable 


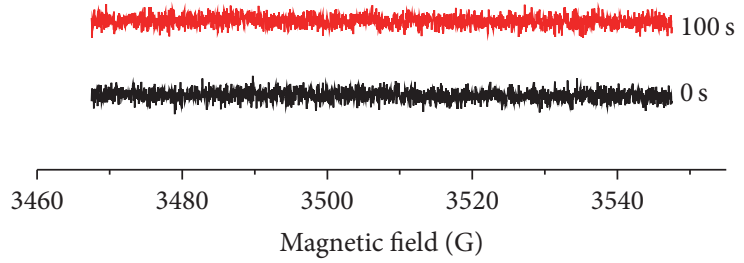

(a)

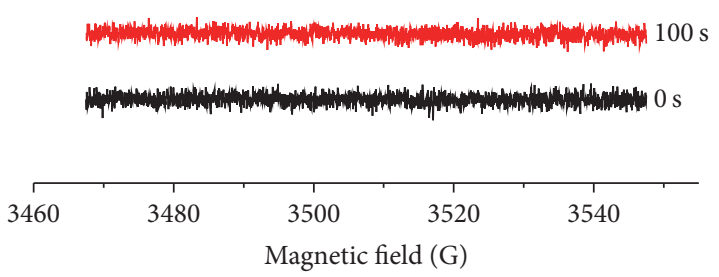

(b)

FIGURE 12: DMPO spin-trapping EPR spectra in aqueous (a) and ethanol (b) solution containing AR1 (0.05 mM) ([OMC-CoTAPc-EPTAC] = $0.4 \mathrm{~g} / \mathrm{L},\left[\mathrm{H}_{2} \mathrm{O}_{2}\right]=15 \mathrm{mM}$, and $\left.[\mathrm{DMPO}]=20 \mathrm{mM}\right)$.

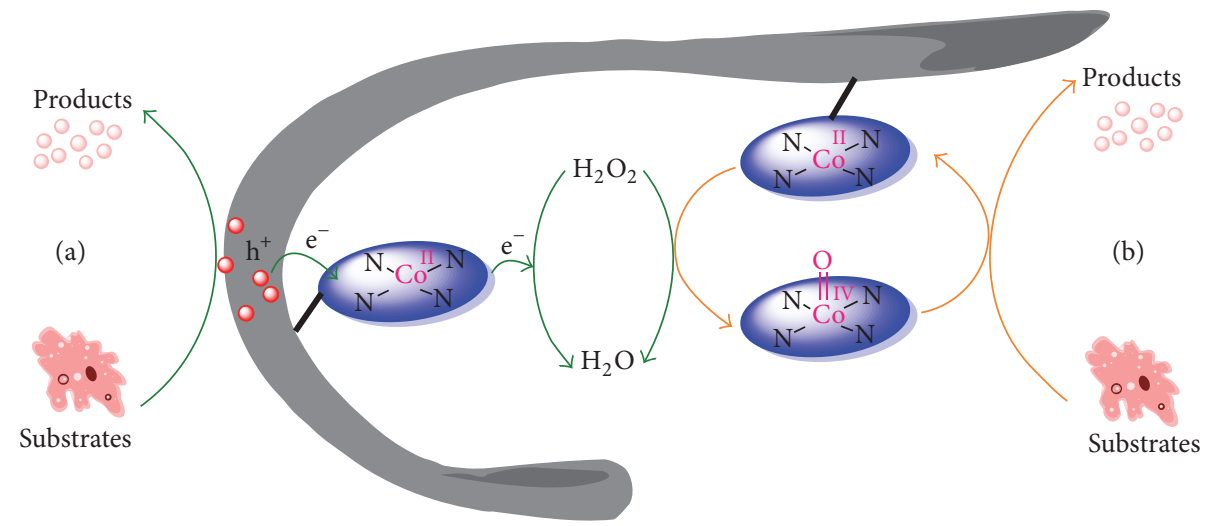

FIGURE 13: The possible pathways for the oxidation of AR1 in this catalytic system ((a) generation of holes in OMC; (b) generation of highvalent cobalt-oxo species).

technology for the treatment of organic pollutants in low concentrations.

\section{Competing Interests}

The authors declare that there is no conflict of interests regarding the publication of this paper.

\section{Acknowledgments}

This work was supported by the National Natural Science Foundation of China (no. 51133006), Zhejiang Provincial Natural Science Foundation of China (nos. LY14E030013 and LQ17E030003), and the Public Welfare Technology Application Research Project of Zhejiang Province (no. 2015C33018).

\section{References}

[1] Z. Sun, B. Sun, M. Qiao et al., "A general chelate-assisted coassembly to metallic nanoparticles-incorporated ordered mesoporous carbon catalysts for Fischer-Tropsch synthesis," Journal of the American Chemical Society, vol. 134, no. 42, pp. 1765317660, 2012.

[2] M. Xie, K. Fang, Y. Shen et al., "Catalytic hydroxylation enables phenol to efficient assembly of ordered mesoporous carbon under highly acidic conditions," Microporous and Mesoporous Materials, vol. 223, pp. 114-120, 2016.

[3] P. Srinivasu, S. P. Singh, A. Islam, and L. Han, "Solar energy conversion by dye-sensitized photovoltaic cells using high surface area mesoporous carbon counter electrode," Advances in OptoElectronics, vol. 2011, Article ID 504217, 4 pages, 2011.

[4] Y. Song, J. Yang, K. Wang et al., "In-situ synthesis of graphene/ nitrogen-doped ordered mesoporous carbon nanosheet for supercapacitor application," Carbon, vol. 96, pp. 955-964, 2016.

[5] S. Tanaka, H. Fujimoto, J. F. M. Denayer, M. Miyamoto, Y. Oumi, and Y. Miyake, "Surface modification of softlated ordered mesoporous carbon for electrochemical supercapacitors," Microporous and Mesoporous Materials, vol. 217, pp. 141-149, 2015.

[6] A. Corma, "From microporous to mesoporous molecular sieve materials and their use in catalysis," Chemical Reviews, vol. 97, no. 6, pp. 2373-2419, 1997.

[7] X. Zhuang, Y. Wan, C. Feng, Y. Shen, and D. Zhao, "Highly efficient adsorption of bulky dye molecules in wastewater on ordered mesoporous carbons," Chemistry of Materials, vol. 21, no. 4, pp. 706-716, 2009.

[8] C. Vix-Guterl, E. Frackowiak, K. Jurewicz, M. Friebe, J. Parmentier, and F. Béguin, "Electrochemical energy storage in ordered porous carbon materials," Carbon, vol. 43, no. 6, pp. 1293-1302, 2005.

[9] A. Taguchi and F. Schüth, "Ordered mesoporous materials in catalysis," Microporous and Mesoporous Materials, vol. 77, no. 1, pp. 1-45, 2005.

[10] P. Simon and Y. Gogotsi, "Materials for electrochemical capacitors," Nature Materials, vol. 7, no. 11, pp. 845-854, 2008.

[11] M. Muhammad, M. Ali Khan, and T. S. Y. Choong, "Adsorptive separation studies of $\beta$-carotene from methyl ester using mesoporous carbon coated monolith," Journal of Chemistry, vol. 2013, Article ID 235836, 6 pages, 2013. 
[12] P. Srinivasu, "Investigation on the textural properties tuning of ordered mesoporous carbons with an excellent electrochemical performance," Advances in OptoElectronics, vol. 2011, Article ID 615164, 4 pages, 2011.

[13] Y.-S. Hu, P. Adelhelm, B. M. Smarsly, S. Hore, M. Antonietti, and J. Maier, "Synthesis of hierarchically porous carbon monoliths with highly ordered microstructure and their application in rechargeable lithium batteries with high-rate capability," Advanced Functional Materials, vol. 17, no. 12, pp. 1873-1878, 2007.

[14] J.-B. Park, J. Lee, C. S. Yoon, and Y.-K. Sun, "Ordered mesoporous carbon electrodes for $\mathrm{Li}^{-} \mathrm{O}_{2}$ batteries," ACS Applied Materials and Interfaces, vol. 5, no. 24, pp. 13426-13431, 2013.

[15] A. Rezaeifard, M. Jafarpour, A. Naeimi, and M. Salimi, "Efficient and highly selective aqueous oxidation of alcohols and sulfides catalyzed by reusable hydrophobic copper (II) phthalocyanine," Inorganic Chemistry Communications, vol. 15, pp. 230-234, 2012.

[16] Z. Sun, L. Jin, S. He et al., "A structured catalyst based on cobalt phthalocyanine/calcined Mg-Al hydrotalcite film for the oxidation of mercaptan," Green Chemistry, vol. 14, no. 7, pp. 1909-1916, 2012.

[17] R. L. Arechederra, K. Artyushkova, P. Atanassov, and S. D. Minteer, "Growth of phthalocyanine doped and undoped nanotubes using mild synthesis conditions for development of novel oxygen reduction catalysts," ACS Applied Materials and Interfaces, vol. 2, no. 11, pp. 3295-3302, 2010.

[18] J. H. Zagal, S. Griveau, J. F. Silva, T. Nyokong, and F. Bedioui, "Metallophthalocyanine-based molecular materials as catalysts for electrochemical reactions," Coordination Chemistry Reviews, vol. 254, no. 23-24, pp. 2755-2791, 2010.

[19] A. B. Sorokin, "Phthalocyanine metal complexes in catalysis," Chemical Reviews, vol. 113, no. 10, pp. 8152-8191, 2013.

[20] B. Basu, S. Satapathy, and A. K. Bhatnagar, "Merox and related metal phthalocyanine catalyzed oxidation processes," Catalysis Reviews-Science and Engineering, vol. 35, no. 4, pp. 571-609, 1993.

[21] B. Meunier and A. Sorokin, "Oxidation of pollutants catalyzed by metallophthalocyanines," Accounts of Chemical Research, vol. 30, no. 11, pp. 470-476, 1997.

[22] A. Hadasch, A. Sorokin, A. Rabion, and B. Meunier, "Sequential addition of $\mathrm{H}_{2} \mathrm{O}_{2}, \mathrm{pH}$ and solvent effects as key factors in the oxidation of 2,4,6-trichlorophenol catalyzed by iron tetrasulfophthalocyanine," New Journal of Chemistry, vol. 22, no. 1, pp. 45-51, 1998.

[23] M. Zhang, C. Shao, Z. Guo et al., "Highly efficient decomposition of organic dye by aqueous-solid phase transfer and in situ photocatalysis using hierarchical copper phthalocyanine hollow spheres," ACS Applied Materials \& Interfaces, vol. 3, no. 7, pp. 2573-2578, 2011.

[24] X. Tao, W. Ma, T. Zhang, and J. Zhao, "Efficient photooxidative degradation of organic compounds in the presence of iron tetrasulfophthalocyanine under visible light irradiation," Angewandte Chemie-International Edition, vol. 40, no. 16, pp. 30143016, 2001.

[25] W. Chen, W. Lu, Y. Yao, and M. Xu, "Highly efficient decomposition of organic dyes by aqueous-fiber phase transfer and in situ catalytic oxidation using fiber-supported cobalt phthalocyanine," Environmental Science and Technology, vol. 41, no. 17, pp. 6240-6245, 2007.
[26] T. Mugadza and T. Nyokong, "Synthesis and characterization of electrocatalytic conjugates of tetraamino cobalt (II) phthalocyanine and single wall carbon nanotubes," Electrochimica Acta, vol. 54, no. 26, pp. 6347-6353, 2009.

[27] J. Hu, H. Liu, L. Wang et al., "Electronic properties of carbon nanotubes linked covalently with iron phthalocyanine to determine the formation of high-valent iron intermediates or hydroxyl radicals," Carbon, vol. 100, pp. 408-416, 2016.

[28] W. Lu, N. Li, W. Chen, and Y. Yao, "The role of multiwalled carbon nanotubes in enhancing the catalytic activity of cobalt tetraaminophthalocyanine for oxidation of conjugated dyes," Carbon, vol. 47, no. 14, pp. 3337-3345, 2009.

[29] W. Lu, N. Li, S. Bao, W. Chen, and Y. Yao, "The coupling of metallophthalocyanine with carbon nanotubes to produce a nanomaterial-based catalyst for reaction-controlled interfacial catalysis," Carbon, vol. 49, no. 5, pp. 1699-1709, 2011.

[30] W. Lu, W. Chen, N. Li, M. Xu, and Y. Yao, "Oxidative removal of 4-nitrophenol using activated carbon fiber and hydrogen peroxide to enhance reactivity of metallophthalocyanine," Applied Catalysis B: Environmental, vol. 87, no. 3-4, pp. 146-151, 2009.

[31] X. Wang, C. Liang, and S. Dai, "Facile synthesis of ordered mesoporous carbons with high thermal stability by selfassembly of resorcinol-formaldehyde and block copolymers under highly acidic conditions," Langmuir, vol. 24, no. 14, pp. 7500-7505, 2008.

[32] B. N. Achar, G. M. Fohlen, J. A. Parker, and J. Keshavayya, "Synthesis and structural studies of metal(II) 4,9,16,23phthalocyanine tetraamines," Polyhedron, vol. 6, no. 6, pp. 1463-1467, 1987.

[33] N. Li, W. Lu, K. Pei, and W. Chen, "Interfacial peroxidase-like catalytic activity of surface-immobilized cobalt phthalocyanine on multiwall carbon nanotubes," RSC Advances, vol. 5, no. 13, pp. 9374-9380, 2015.

[34] M. S. Hamdy, A. Ramanathan, T. Maschmeyer, U. Hanefeld, and J. C. Jansen, "Co-TUD-1: a ketone-selective catalyst for cyclohexane oxidation," Chemistry-A European Journal, vol. 12, no. 6, pp. 1782-1789, 2006.

[35] M. S. Hamdy and G. Mul, "Synthesis, characterization and catalytic performance of Mo-TUD-1 catalysts in epoxidation of cyclohexene," Catalysis Science \& Technology, vol. 2, no. 9, pp. 1894-1900, 2012.

[36] G. Imran, M. P. Pachamuthu, R. Maheswari, A. Ramanathan, and S. J. Sardhar Basha, "Catalytic activity of MnTUD-1 for liquid phase oxidation of ethylbenzene with tert-butyl hydroperoxide," Journal of Porous Materials, vol. 19, no. 5, pp. 677-682, 2012.

[37] F. Su, X. S. Zhao, Y. Wang, L. Wang, and J. Y. Lee, "Hollow carbon spheres with a controllable shell structure," Journal of Materials Chemistry, vol. 16, no. 45, pp. 4413-4419, 2006.

[38] N. Li, W. Lu, K. Pei, Y. Yao, and W. Chen, "Orderedmesoporous-carbon-bonded cobalt phthalocyanine: a bioinspired catalytic system for controllable hydrogen peroxide activation," ACS Applied Materials and Interfaces, vol. 6, no. 8, pp. 5869-5876, 2014.

[39] C. P. Huang, Y. F. Huang, H. P. Cheng, and Y. H. Huang, "Kinetic study of an immobilized iron oxide for catalytic degradation of azo dye reactive black $B$ with catalytic decomposition of hydrogen peroxide," Catalysis Communications, vol. 10, no. 5, pp. 561-566, 2009.

[40] P. K. Malik, "Oxidation of safranine $\mathrm{T}$ in aqueous solution using Fenton's reagent: involvement of an Fe(III) chelate in 
the catalytic hydrogen peroxide oxidation of safranine T," The Journal of Physical Chemistry A, vol. 108, no. 14, pp. 2675-2681, 2004.

[41] W. C. Ellis, C. T. Tran, R. Roy et al., "Designing green oxidation catalysts for purifying environmental waters," Journal of the American Chemical Society, vol. 132, no. 28, pp. 9774-9781, 2010.

[42] N. Li, W. Lu, K. Pei, Y. Yao, and W. Chen, "Formation of high-valent cobalt-oxo phthalocyanine species in a cellulose matrix for eliminating organic pollutants," Applied Catalysis B: Environmental, vol. 163, pp. 105-112, 2015. 

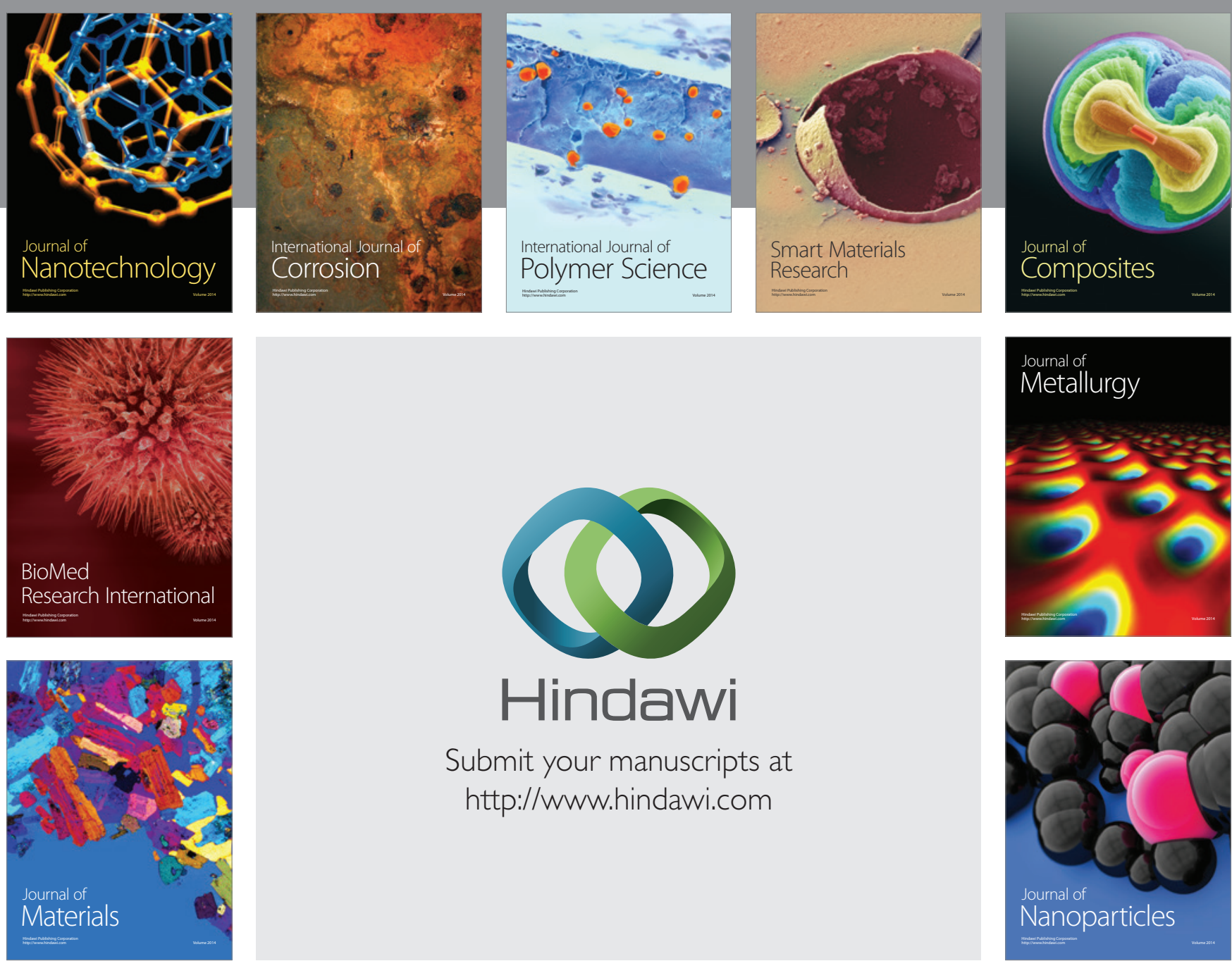

\section{Hindawi}

Submit your manuscripts at

http://www.hindawi.com

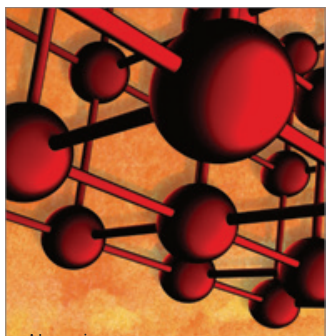

Materials Science and Engineering
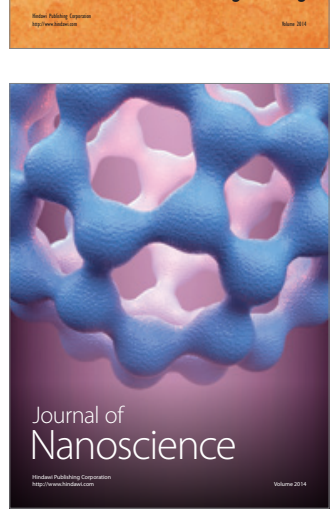
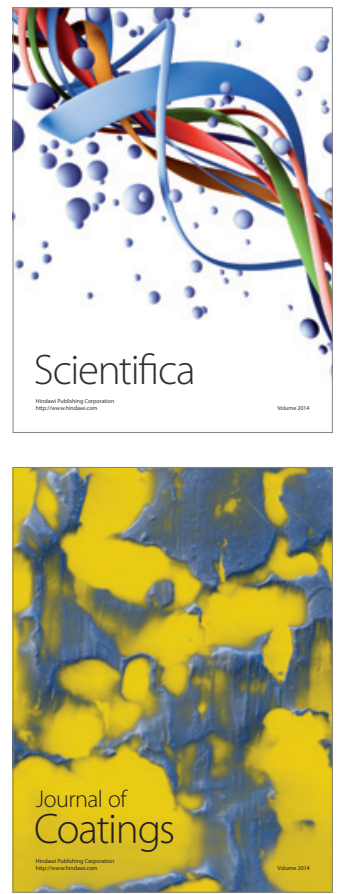
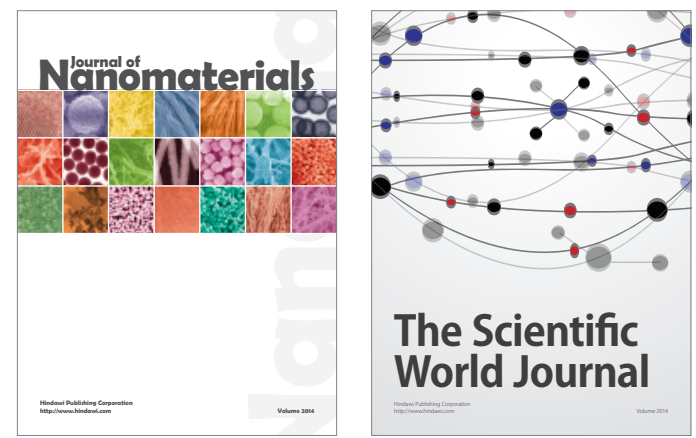

The Scientific World Journal
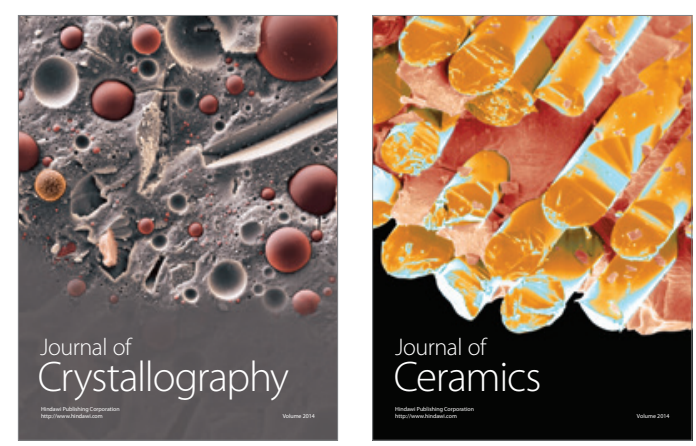
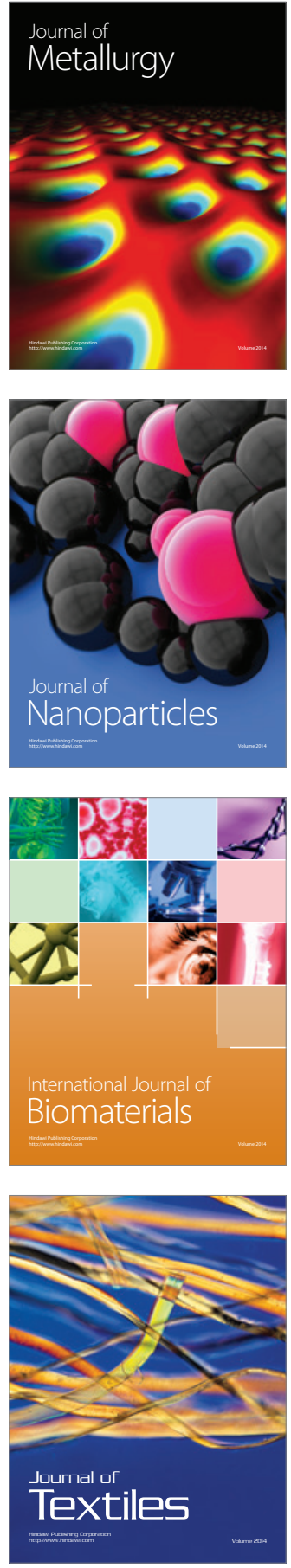